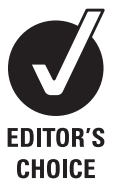

- An additional appendix is published online only. To view this file please visit the journal online (http://thorax.bmj.com).

${ }^{1}$ Department of Paediatric Oncology, Emma Children's Hospital/Academic Medical Center, Amsterdam, The Netherlands

${ }^{2}$ Department of Pulmonology, Academic Medical Center, Amsterdam, The Netherlands ${ }^{3}$ Department of Respiratory Medicine, OLVG Hospital, Amsterdam, The Netherlands ${ }^{4}$ Department of Medical Oncology, Academic Medical Center, Amsterdam, The Netherlands

${ }^{5}$ Department of Pulmonology, St Franciscus Hospital, Rotterdam, The Netherlands

${ }^{6}$ Department of Radiation Oncology, Academic Medical Center, Amsterdam, The Netherlands

${ }^{7}$ Department of Paediatric Surgery, Emma Children's Hospital/Academic Medical Center, Amsterdam, The Netherlands

\section{Correspondence to}

Renée L Mulder, Department of

Paediatric Oncology, F8-207.

Emma Children's

Hospital/Academic Medical

Center, PO Box 22660, 1100 DD

Amsterdam, The Netherlands;

r.l.mulder@amc.uva.nl

RLM and NMT contributed equally to this work.

Received 21 December 2010 Accepted 30 June 2011 Published Online First

29 July 2011

\title{
Pulmonary function impairment measured by pulmonary function tests in long-term survivors of childhood cancer
}

\author{
Renée L Mulder, ${ }^{1}$ Nienke M Thönissen, ${ }^{2,3}$ Helena J H van der Pal, ${ }^{1,4}$ Paul Bresser, ${ }^{2,3}$ \\ Wessel Hanselaar, ${ }^{5}$ Caro C E Koning, ${ }^{6}$ Foppe Oldenburger, ${ }^{6}$ Hugo A Heij, ${ }^{7}$ \\ Huib N Caron, ${ }^{1}$ Leontien C M Kremer ${ }^{1}$
}

\begin{abstract}
Background Childhood cancer survivors (CCSs) have an increased risk of morbidity and mortality. The prevalence and risk factors of pulmonary function impairment were investigated in a large cohort of CCSs treated with potentially pulmotoxic therapy with a minimal follow-up of 5 years after diagnosis.
\end{abstract}

Methods The study cohort consisted of all adult 5-year CCSs who were treated with bleomycin, pulmonary radiotherapy and/or pulmonary surgery in the Emma Children's Hospital/Academic Medical Center between 1966 and 1996. Pulmonary function tests were performed to diagnose obstructive and restrictive pulmonary function impairment, and diffusion capacity impairment.

Results The study population consisted of 220 out of 248 eligible CCSs, of whom 193 (87.7\%) had performed a pulmonary function test at a median follow-up of 18 years after diagnosis. 85 (44.0\%) out of 193 CCSs developed a pulmonary function impairment. Pulmonary function impairments occurred in all treatment groups. Most prevalent were restrictive pulmonary function impairment (17.6\%) and a decreased carbon monoxide diffusion capacity (39.9\%). Multivariate logistic regression models showed that, compared with bleomycin treatment only, treatment with radiotherapy, radiotherapy combined with bleomycin and radiotherapy combined with surgery were associated with the highest risk of pulmonary function impairment.

Conclusions The prevalence of pulmonary function impairment in long-term adult CCSs who received potentially pulmotoxic therapy is high. Bleomycin, pulmonary radiotherapy and pulmonary surgery are all associated with pulmonary function impairment. Pulmonary radiotherapy, especially in combination with bleomycin or surgery, is the most important risk factor. This emphasises the need for adequate counselling and follow-up for this patient population.

\section{INTRODUCTION}

Due to improvement of cancer treatment over the last 40 years there has been a dramatic increase in survival of childhood malignancies. At present, the 5-year survival reaches $80 \%$ in developed countries. ${ }^{12}$ Unfortunately, the improved prognosis has allowed the occurrence of late, treatment-related complications. $^{3} 4$

Childhood cancer survivors (CCSs) treated with bleomycin, pulmonary radiotherapy and/or

\section{Key messages}

What is the key question?

- What are the prevalence and risk factors of pulmonary function impairment in a large cohort of childhood cancer survivors treated with potentially pulmotoxic therapy with a minimal follow-up of 5 years after diagnosis?

\section{What is the bottom line?}

- To give more insight into the long-term risk of pulmonary function impairment after treatment for childhood cancer in order to establish adequate follow-up protocols.

\section{Why read on?}

- This paper gives new insight into the risk of pulmonary function impairment in long-term survivors of childhood cancer treated with potentially pulmotoxic therapy.

pulmonary surgery are at risk for pulmonary function impairment. The lungs are liable to suffer from the adverse effects of radiotherapy. Abnormalities in pulmonary function have been observed in patients after low doses of pulmonary irradiation. Apart from bleomycin, which is commonly recognised as a cause of chemotherapy-related pulmonary toxicity, busulphan, carmustine, lomustine, melphalan and cyclophosphamide are also reported to cause pulmonary function impairment. ${ }^{5-7}$

Treatment-related pulmonary function impairment may cause symptoms of chronic cough, recurrent respiratory tract infections, shortness of breath and/or exercise intolerance, and may involve early and late effects. Late pulmonary function impairment includes obstructive and restrictive pulmonary disease, and diffusion capacity impairments. ${ }^{5-7}$ This may affect quality of life and can lead to increased risks of premature morbidity and mortality. 8

Several studies have evaluated the prevalence of pulmonary function impairment in CCSs. ${ }^{9-17}$ The reported prevalence varied widely. Most studies, however, had limited sample size, short follow-up duration and/or studied specific patient populations. The Childhood Cancer Survivor Study showed that the risk of pulmonary complications 
detected by self-reports is more than three times higher in childhood cancer survivors (CCSs) than in their siblings. ${ }^{18}$ However, in that study, no pulmonary function tests were performed. Until now, no studies have investigated the risk of pulmonary function impairment measured by pulmonary function tests in a large cohort of long-term adult CCSs.

In order to establish adequate follow-up protocols for CCSs, it is important to estimate the risk of pulmonary function impairment. Therefore, the aim of this study was to evaluate the prevalence and risk factors of pulmonary function impairment measured by pulmonary function tests in a large cohort of adult CCSs treated with potentially pulmotoxic therapy with a minimal follow-up of 5 years after diagnosis.

\section{METHODS}

\section{Study population}

In 1996, the Late Effects Outpatient Clinic for CCSs was established in the Emma Children's Hospital/Academic Medical Center (EKZ/AMC). All 5-year CCSs were identified using the Childhood Cancer Registry, which was established in 1966 and contains data on all patients treated for childhood cancer in the EKZ/AMC regarding diagnosis, treatment and follow-up. All CCSs surviving for at least 5 years were invited to participate and enrolled into prospective follow-up study protocols tailored to previous diagnosis and treatment. CCSs treated with bleomycin, pulmonary radiotherapy or pulmonary surgery were enrolled in the pulmotoxic screening protocol. To be eligible for this study, patients had to meet the following criteria: (1) diagnosed between January 1966 and January 1996 and treated for a primary malignancy; (2) aged <18 years at diagnosis; (3) treated mainly in the EKZ/AMC; (4) survived for at least 5 years after diagnosis at 1 January 2007; (5) qualified for the pulmotoxic screening protocol; and (6) aged 18 years or older at pulmonary function testing.

\section{Follow-up and data collection}

All CCSs who visited the Late Effects Outpatient Clinic between January 1996 and January 2009 received a full medical assessment according to the previously mentioned protocols. This medical assessment consisted of a medical history, physical examination and additional risk-based diagnostic tests and counselling. The pulmotoxic screening protocol included pulmonary function testing. For each CCS information was recorded concerning patient and treatment characteristics and pulmonary function testing.

\section{Definition of pulmonary function impairment}

For the evaluation of pulmonary function impairment we assessed the first complete pulmonary function test performed at least 5 years after diagnosis. We evaluated the total lung capacity (TLC), forced vital capacity (FVC), forced expiratory volume in $1 \mathrm{~s}\left(\mathrm{FEV}_{1}\right), \mathrm{FEV}_{1} /$ maximal vital capacity $\left(\mathrm{VC}_{\max }\right)$ ratio, carbon monoxide diffusion capacity corrected for haemoglobin concentration $\left(\mathrm{D}_{\mathrm{L}} \mathrm{CO}\right)$ and carbon monoxide diffusion capacity corrected for alveolar volume, also called the carbon monoxide transfer coefficient (KCO).

According to the Global Initiative for Chronic Obstructive Lung Disease (GOLD) criteria, ${ }^{19}$ obstructive pulmonary function impairment was defined as an $\mathrm{FEV}_{1} / \mathrm{VC}_{\max }$ ratio $<0.70$ and $\mathrm{FEV}_{1}<80 \%$ of the predicted value. The disease severity was graded as follows: (0) no impairment, $\mathrm{FEV}_{1} / \mathrm{VC}_{\max }$ ratio $>0.70$ and $\mathrm{FEV}_{1}>80 \%$ of predicted value; (1) mild impairment, $\mathrm{FEV}_{1} / \mathrm{VC}_{\max }$ ratio $<0.70$ and $\mathrm{FEV}_{1} \geq 80 \%$ of predicted value; (2) moderate impairment, $\mathrm{FEV}_{1} / \mathrm{VC}_{\max }$ ratio $<0.70$ and
$\mathrm{FEV}_{1}<80-50 \%$ of predicted value; (3) severe impairment, $\mathrm{FEV}_{1} / \mathrm{VC}_{\max }$ ratio $<0.70$ and $\mathrm{FEV}_{1}<50-30 \%$ predicted value; and (4) very severe impairment, $\mathrm{FEV}_{1} / \mathrm{VC}_{\max }$ ratio $<0.70$ and $\mathrm{FEV}_{1}<30 \%$ of predicted value or $\mathrm{FEV}_{1} / \mathrm{VC}_{\max }$ ratio $<0.70$ and $\mathrm{FEV}_{1}<50 \%$ of predicted value with chronic respiratory failure.

Restrictive pulmonary function impairment and diffusion capacity impairment were defined according to the Common Terminology Criteria for Adverse Events version 3.0 (CTCAE v3.0). ${ }^{20}$ Restrictive pulmonary function impairment was defined as TLC $<75 \%$ of the predicted value. If no TLC measurements were available, it was defined as FVC $<75 \%$ of the predicted value with a normal $\mathrm{FEV}_{1} / \mathrm{VC}_{\max }$ ratio. Diffusion capacity impairment was measured using the $\mathrm{D}_{\mathrm{L}} \mathrm{CO}$ and $\mathrm{KCO}$ and was defined as $<75 \%$ of the predicted value. The disease severity of restrictive pulmonary function impairment and diffusion capacity impairment was graded from 0 to 5: (0) no impairment, $>90 \%$ of predicted value; (1) mild impairment, $90-75 \%$ of predicted value; (2) moderate impairment, $<75-50 \%$ of predicted value; (3) severe and undesirable impairment, $<50-25 \%$ of predicted value; (4) life-threatening or disabling impairment, $<25 \%$ of predicted value; and (5) death.

By definition, pulmonary function impairment of grade 2 or higher can be considered abnormal, reflecting physiological impairment. ${ }^{21}$

\section{Statistical analysis}

We performed multivariate logistic regression analyses to evaluate possible risk factors for restrictive pulmonary function impairment and diffusion capacity impairment measured by $\mathrm{D}_{\mathrm{L}} \mathrm{CO}$. Because of small event numbers, it was not possible to evaluate risk factors for obstructive pulmonary function impairment and diffusion capacity impairment measured by KCO.

We constructed two models in which the following risk factors were evaluated: sex, age at diagnosis, follow-up duration and treatment with bleomycin, pulmonary radiotherapy, pulmonary surgery and high-dose cyclophosphamide (high-dose was defined as $\geq 1 \mathrm{~g} / \mathrm{m}^{2}$ per course or a total cumulative dose of $\geq 10 \mathrm{~g} / \mathrm{m}^{2}$ ). In model 1 , potentially pulmotoxic treatments were modelled as independent risk factors consisting of yes/no variables, whereas in model 2 these treatments were modelled as a categorical risk factor consisting of mutually exclusive groups.

Analyses were performed using statistical software SPSS for Windows 15.0.1 (release 2007).

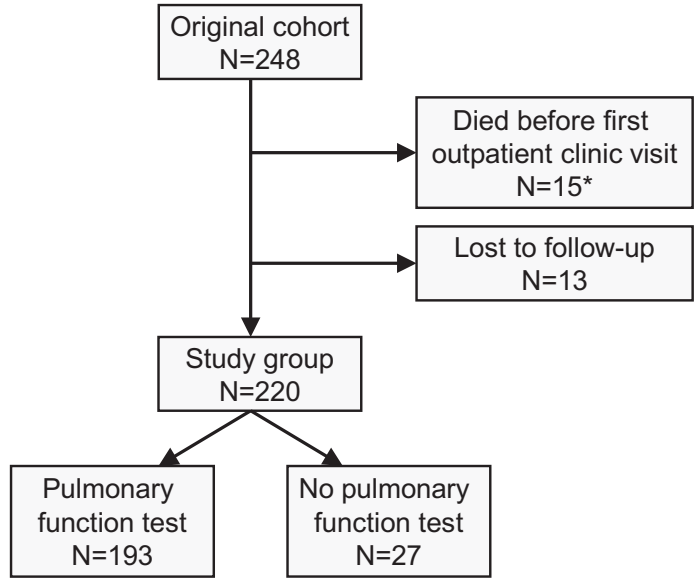

Figure 1 Flowchart of childhood cancer survivors included in this study. * None of the 15 childhood cancer survivors who died before they could visit the outpatient clinic died because of pulmonary disease. 
Table 1 Clinical characteristics of childhood cancer survivors treated with potentially pulmotoxic therapy

\begin{tabular}{|c|c|c|c|}
\hline Characteristic & Original cohort $\mathbf{n}(\%)$ & Study group $\mathbf{n}(\%)$ & Follow-up group* n (\%) \\
\hline No. of childhood cancer survivors & 248 & 220 & 193 \\
\hline \multicolumn{4}{|l|}{ Sex } \\
\hline Male & $136(54.8)$ & $117(53.2)$ & 103 (53.4) \\
\hline Female & $112(45.2)$ & $103(46.8)$ & $90(46.6)$ \\
\hline \multicolumn{4}{|l|}{ Primary childhood cancer diagnosis } \\
\hline Leukaemia & $34(13.7)$ & $28(12.7)$ & $23(11.9)$ \\
\hline Lymphoma & $131(52.8)$ & $121(55.0)$ & $110(57.0)$ \\
\hline Hodgkin & $62(25.0)$ & $57(25.9)$ & $52(26.9)$ \\
\hline Non-Hodgkin & $69(27.8)$ & $64(29.1)$ & $58(30.1)$ \\
\hline Renal tumour & $19(7.7)$ & $16(7.3)$ & $13(6.7)$ \\
\hline Brain/CNS tumour & $0(0.0)$ & $0(0.0)$ & $0(0.0)$ \\
\hline Bone tumour & $30(12.1)$ & $23(10.4)$ & $20(10.4)$ \\
\hline Soft tissue sarcoma & $6(2.4)$ & $5(2.3)$ & $4(2.1)$ \\
\hline Neuroblastoma & $9(3.6)$ & $9(4.1)$ & $7(3.6)$ \\
\hline Other & $19(7.7)$ & $18(8.2)$ & $16(8.3)$ \\
\hline Age at diagnosis, median (range) years & $10.0(0.0-17.1)$ & $9.9(0.0-17.1)$ & $10.3(0.0-16.8)$ \\
\hline $0-4$ years & $51(20.6)$ & $47(21.4)$ & $34(17.6)$ \\
\hline $5-9$ years & $71(28.6)$ & $65(29.5)$ & $59(30.6)$ \\
\hline $10-14$ years & $100(40.3)$ & $84(38.2)$ & $78(40.4)$ \\
\hline $15-18$ years & $26(10.5)$ & $24(10.9)$ & $22(11.4)$ \\
\hline $\begin{array}{l}\text { Follow-up duration after diagnosis, } \\
\text { median (range) years } \dagger\end{array}$ & NA & NA & $17.9(5.6-36.8)$ \\
\hline $5-9$ years & & & $20(10.4)$ \\
\hline $10-14$ years & & & $45(23.3)$ \\
\hline $15-19$ years & & & $50(25.9)$ \\
\hline $20-24$ years & & & $40(20.7)$ \\
\hline$\geq 25$ years & & & $38(19.7)$ \\
\hline Age at follow-up, median (range) years $\dagger$ & NA & NA & $27.3(18.2-47.0)$ \\
\hline $18-19$ years & & & $22(11.4)$ \\
\hline $20-24$ years & & & $47(24.4)$ \\
\hline $25-29$ years & & & $49(25.4)$ \\
\hline $30-34$ years & & & $40(20.7)$ \\
\hline$\geq 35$ years & & & $35(18.1)$ \\
\hline $\begin{array}{l}\text { Bleomycin, median cumulative dose } \\
\text { (range) } \mathrm{mg} / \mathrm{m}^{2}\end{array}$ & $60.0(6.0-594.0)$ & $60.0(10.0-594.0)$ & $60.0(10.0-594.0)$ \\
\hline Yes & $134(54.0)$ & $124(56.4)$ & $110(57.0)$ \\
\hline No & $114(46.0)$ & $96(43.6)$ & $83(43.0)$ \\
\hline $\begin{array}{l}\text { Radiotherapy, median cumulative dose } \\
\text { (range) Gy }\end{array}$ & $21.0(3.0-60.0)$ & $24.3(3.0-60.0)$ & $23.0(3.0-60.0)$ \\
\hline Yes & $103(41.5)$ & $86(39.1)$ & 79 (40.9) \\
\hline Complete thorax & $20(8.1)$ & $15(6.8)$ & $13(6.7)$ \\
\hline Part of thorax & $30(12.1)$ & $27(12.3)$ & $26(13.5)$ \\
\hline Mediastinum & $33(13.3)$ & $29(13.2)$ & $26(13.5)$ \\
\hline $\mathrm{TBI}$ & $20(8.1)$ & $15(6.8)$ & $14(7.3)$ \\
\hline No & $145(58.5)$ & $134(60.9)$ & $114(59.1)$ \\
\hline \multicolumn{4}{|l|}{ Surgery } \\
\hline Yes & $47(19.0)$ & 40 (18.2) & $32(16.6)$ \\
\hline Metastasectomy unilateral & $14(5.6)$ & $13(5.9)$ & $9(4.7)$ \\
\hline Metastasectomy bilateral & $15(6.0)$ & $12(5.4)$ & $11(5.7)$ \\
\hline Lobectomy & $4(1.6)$ & $1(0.5)$ & $0(0.0)$ \\
\hline Pneumonectomy & $1(0.4)$ & $1(0.5)$ & $1(0.0)$ \\
\hline Thoracic wall resection & $5(2.0)$ & $5(2.3)$ & $5(2.6)$ \\
\hline Other & $8(3.2)$ & $8(3.6)$ & $6(3.1)$ \\
\hline No & $201(81.0)$ & $180(81.8)$ & $161(83.4)$ \\
\hline \multicolumn{4}{|l|}{ Overall treatment } \\
\hline Bleomycin only & $120(48.4)$ & $111(50.5)$ & $98(50.8)$ \\
\hline Radiotherapy only & $70(28.2)$ & $59(26.8)$ & $54(28.0)$ \\
\hline Surgery only & $22(8.9)$ & $20(9.1)$ & $13(6.7)$ \\
\hline Bleomycin with radiotherapy & $11(4.4)$ & $10(4.5)$ & $9(4.7)$ \\
\hline Bleomycin with surgery & $3(1.2)$ & $3(1.4)$ & $3(1.6)$ \\
\hline Radiotherapy with surgery & $22(8.9)$ & $17(7.7)$ & $16(8.3)$ \\
\hline $\begin{array}{l}\text { Bleomycin with radiotherapy and } \\
\text { surgery }\end{array}$ & $0(0.0)$ & $0(0.0)$ & $0(0.0)$ \\
\hline
\end{tabular}


Table 1 Continued

\begin{tabular}{llll}
\hline Characteristic & Original cohort $\mathbf{n}(\%)$ & Study group n (\%) & Follow-up group* n (\%) \\
\hline $\begin{array}{l}\text { Other chemotherapy } \\
\text { HD cyclophosphamide }\end{array}$ & $52(21.0)$ & $47(21.4)$ & $43(22.3)$ \\
Melphalan & $6(2.4)$ & $3(1.4)$ & $3(1.6)$ \\
Busulphan & $0(0.0)$ & $0(0.0)$ & $0(0.0)$ \\
Lomustine & $1(0.4)$ & $0(0.0)$ & $0(0.0)$ \\
$\quad$ Carmustine & $3(1.2)$ & $3(1.4)$ & $3(1.6)$ \\
Other & $231(93.1)$ & $204(92.7)$ & $180(93.3)$ \\
Recurrence of primary tumour & & & \\
Yes & $81(32.7)$ & $64(29.1)$ & $54(28.0)$ \\
No & $167(67.3)$ & $156(70.9)$ & $139(72.0)$ \\
Vital status & & & \\
$\quad$ Living & $229(92.3)$ & $216(98.2)$ & $191(99.0)$ \\
Died & $19(7.7)$ & $4(1.8)$ & $2(1.0)$ \\
\hline
\end{tabular}

*Childhood cancer survivors who performed pulmonary function tests.

†Follow-up duration can only be calculated for childhood cancer survivors who performed pulmonary function tests. CNS, central nervous system; HD, high-dose; TBI, total body irradiation.

\section{RESULTS \\ Study population}

The study population consisted of 220 out of 248 eligible CCSs. Thirteen CCSs of the total potential CCSs who lived at least 5 years from diagnosis were lost to follow-up and 15 CCSs died before the first outpatient clinic visit (none of pulmonary disease) (figure 1). Clinical characteristics of the study population are presented in table 1. Out of 220 CCSs, 193 (87.7\%) performed a pulmonary function test at the age of 18 years or older. The median follow-up time since diagnosis was 17.9 years, ranging from 5.6 to 36.8 years. There were no differences in clinical characteristics between the CCSs in the original cohort, the CCSs in the entire study group and the CCSs in whom pulmonary function tests were performed.

\section{Prevalence of pulmonary function impairment}

Table 2 shows the prevalence of pulmonary function impairment (grade 2 or higher) by therapy subgroup in 193 CCSs with pulmonary function tests. In figure 2 , the severity of pulmonary function impairment is presented. Four CCSs (2.1\%) had an obstructive pulmonary function impairment. The disease severity in these patients was moderate (grade 2). None had a severe obstructive pulmonary function impairment (grade 3-5). Two CCSs (1.0\%) had a mild obstructive pulmonary function impairment (grade 1).

Thirty-four CCSs (17.6\%) had a restrictive pulmonary function impairment. The highest prevalence was seen in CCSs treated with the combination of radiotherapy and surgery; that is, $10(62.5 \%)$ out of 16 . The severity was moderate (grade 2 ) in 31 patients (16.1\%) and severe (grade 3 ) in 3 patients $(1.6 \%)$. None had restrictive pulmonary function impairment of grade 4 or 5. Moreover, 78 CCSs (40.4\%) had grade 1 restrictive pulmonary function impairment.

For 188 (97.4\%) out of 193 CCSs, carbon monoxide diffusion capacity measurements were available. The prevalence of a decreased $\mathrm{D}_{\mathrm{L}} \mathrm{CO}$ was $39.9 \%$ (75 CCSs). The highest prevalence was seen in CCSs treated with the combination of radiotherapy and surgery; that is, 11 (73.3\%) out of 15 . In 73 CCSs (38.8\%) the $\mathrm{D}_{\mathrm{L}} \mathrm{CO}$ was moderately decreased (grade 2) and in 2 CCSs $(1.1 \%)$ severely decreased (grade 3 ). None had a diffusion capacity impairment of grade 4 or 5 . Furthermore, in 78 patients $(41.5 \%)$, the $\mathrm{D}_{\mathrm{L}} \mathrm{CO}$ was mildly decreased (grade 1 ).

Eight CCSs (4.3\%) had a decreased carbon monoxide diffusion capacity corrected for alveolar volume $(\mathrm{KCO})$, of whom four were treated with bleomycin only and four with radiotherapy only. The disease severity in these patients was moderate (grade 2). None had severe reductions in KCO (grade $3-5$ ). In addition, 57 CCSs (30.3\%) had a mild diffusion capacity impairment (grade 1).

In total, 85 (44.0\%) out of 193 CCSs had pulmonary function impairments (grade 2 or higher) defined as either obstructive or restrictive pulmonary function impairment, decreased $\mathrm{D}_{\mathrm{L}} \mathrm{CO}$ or decreased KCO. Pulmonary function impairments occurred in all treatment groups, with a prevalence ranging from $28.6 \%$ in CCSs treated with bleomycin only up to $81.3 \%$ in CCSs treated with the combination of radiotherapy and surgery. Twenty-eight CCSs $(14.5 \%)$ had both restrictive pulmonary function impairment and a decreased $\mathrm{D}_{\mathrm{L}} \mathrm{CO}$, of whom $1(0.5 \%)$ had a decreased KCO as well. Moreover, 1 CCS (0.5\%) had obstructive pulmonary function impairment and a decreased $\mathrm{D}_{\mathrm{L}} \mathrm{CO}$. In addition, 6 CCSs $(3.1 \%)$ had both a decreased $\mathrm{D}_{\mathrm{L}} \mathrm{CO}$ and $\mathrm{KCO}$ without obstructive or restrictive pulmonary function impairment. Onehundred and forty-one CCSs (73.1\%) had one or more mild pulmonary function impairments (grade 1).

\section{Risk factors for pulmonary function impairment}

The results of the multivariate logistic regression analyses of the risk factors for pulmonary function impairment are presented in table 3. Please note that all CCSs were treated with potentially pulmotoxic therapy.

According to model 1, pulmonary radiotherapy (OR 12.87; 95\% CI 3.37 to 49.08 ) and pulmonary surgery (OR 3.79; 95\% CI 1.25 to 11.50$)$ were significantly associated with restrictive pulmonary function impairment. As shown in model 2, radiotherapy only (OR 6.99; 95\% CI 2.27 to 21.54), bleomycin combined with radiotherapy (OR 9.41; 95\% CI 1.71 to 51.86 ) and radiotherapy combined with surgery (OR 33.44; $95 \% \mathrm{CI}$ 7.81 to 143.09 ) were significantly associated with restrictive pulmonary function impairment, compared with bleomycin only.

Regarding diffusion capacity impairment, pulmonary radiotherapy (OR 5.84; 95\% CI, 1.88 to 18.14) was significantly associated with a decreased $\mathrm{D}_{\mathrm{L}} \mathrm{CO}$ (model 1). In addition, model 2 showed that radiotherapy only (OR 2.85; 95\% CI 1.32 to 6.19), bleomycin combined with radiotherapy (OR 6.17; 95\% CI 1.37 to 27.84) and radiotherapy combined with surgery (OR 5.98; $95 \%$ CI 1.64 to 21.81 ) were significantly associated with a reduced $\mathrm{D}_{\mathrm{L}} \mathrm{CO}$, compared with bleomycin only. 


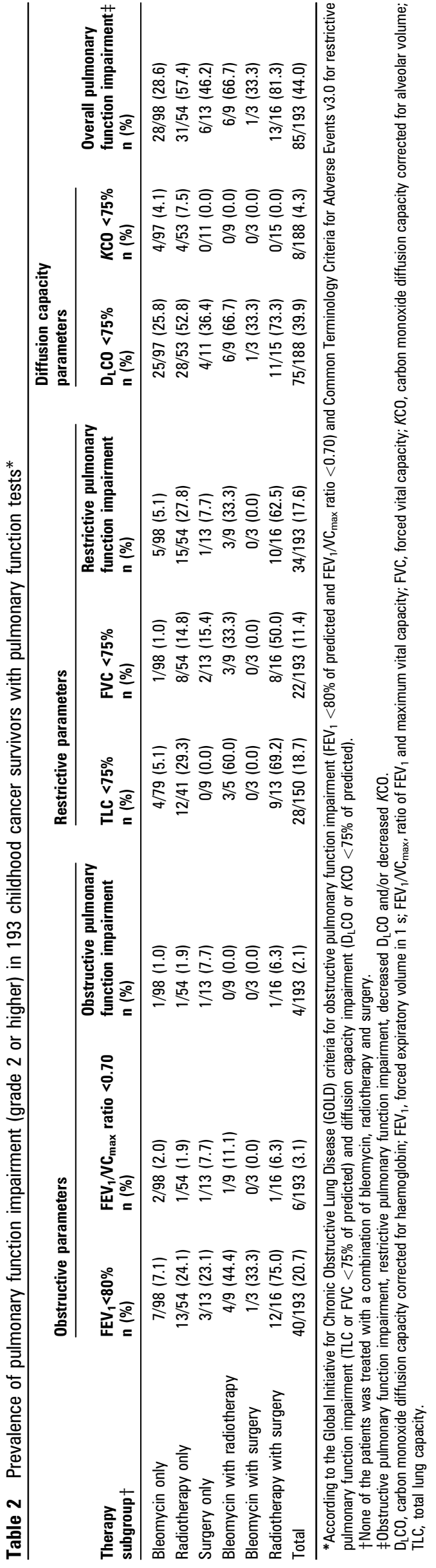

Since our study population consisted of CCSs treated in different eras, we conducted a subgroup analysis on CCSs treated before and after 1985 to evaluate whether the results may have been biased by a cohort effect. The logistic regression analyses on both groups, however, showed similar results to the analyses on the whole group of CCSs (see online appendix 1).

\section{DISCUSSION}

At a median follow-up of 18 years, $44 \%$ of the adult CCSs treated with pulmotoxic therapy developed pulmonary function impairments. In particular, restrictive pulmonary function impairment and diffusion capacity impairment were prevalent. In contrast, obstructive pulmonary function impairment was observed in only a few CCSs. Bleomycin, pulmonary radiotherapy and pulmonary surgery were all associated with pulmonary function impairment. Compared with bleomycin only, treatment with radiotherapy only, radiotherapy combined with bleomycin and radiotherapy combined with surgery were associated with the highest risk of pulmonary function impairment.

This is the first large cohort study investigating long-term pulmonary function impairment measured by pulmonary function tests in CCSs treated with potentially pulmotoxic therapy. Few studies have focused on long-term pulmonary function impairment in CCSs treated with a combination of bleomycin, pulmonary radiotherapy and/or surgery. Oguz et a $1^{10}$ showed that out of 75 childhood lymphoma survivors treated with chemotherapy, including bleomycin and/or thoracic radiotherapy, $13 \%$ had pulmonary function abnormalities at a median follow-up of 5 years after diagnosis. In addition, Bossi et $a l^{11}$ demonstrated that at a median follow-up of 6 years $48 \%$ out of 27 childhood lymphoma survivors treated with mediastinal radiotherapy and/or chemotherapy, including bleomycin, developed pulmonary function impairments. Moreover, Kaplan et $a l^{17}$ found an even higher prevalence of pulmonary function impairments (87\%) in 17 survivors of childhood rhabdomyosarcoma treated with bleomycin, of whom two also received pulmonary radiotherapy and surgery. In agreement with our results, these studies showed that pulmonary function impairment mainly involved restrictive pulmonary function impairment and diffusion capacity impairment rather than obstructive pulmonary function impairment. However, the results of these studies must be interpreted with caution, since sample sizes were limited and follow-up durations relatively short. Here, we have followed a large and nearly complete cohort of 193 CCSs over a median follow-up of 18 years.

We showed that pulmonary radiotherapy, especially in combination with bleomycin or surgery, is the most important risk factor for pulmonary function impairment. Although previous studies also showed that pulmonary radiotherapy increases the risk of pulmonary function impairment, ${ }^{11} 132223$ this is the first study indicating that the combination of radiotherapy with bleomycin or surgery is associated with an even higher risk.

It has been suggested that besides bleomycin, other chemotherapeutics, such as high-dose cyclophosphamide, busulphan, melphalan, lomustine, carmustine and methotrexate, are also associated with pulmonary function impairment. ${ }^{5}{ }^{6}$ However, due to the low methodological quality of these studies, the evidence is not convincing. In multivariate logistic regression analyses we did not find that high-dose cyclophosphamide was associated with an additional increased risk of late pulmonary function impairment. Because only six CCSs were treated with melphalan and carmustine and none with busulphan or 


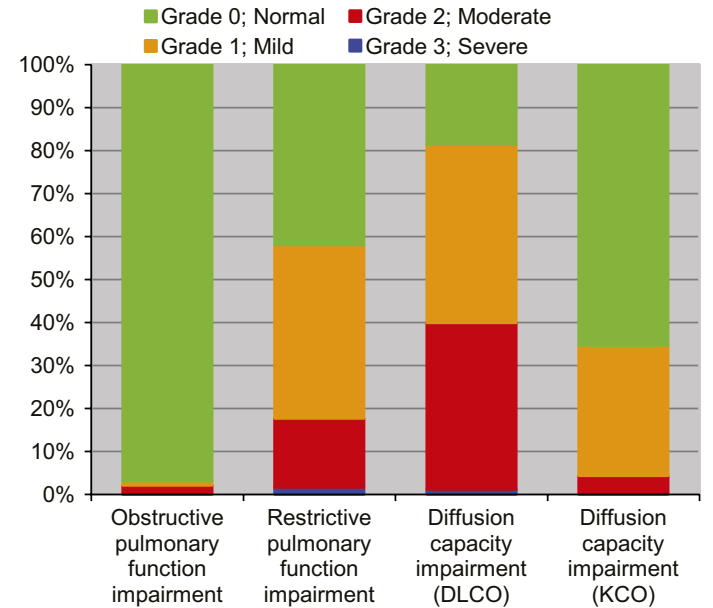

Figure 2 Severity of pulmonary function impairment in 193 childhood cancer survivors with pulmonary function tests.

lomustine, it was impossible to investigate whether these chemotherapeutics were associated with an additional increased risk of pulmonary function impairment. However, since we only included patients treated with bleomycin, pulmonary radiotherapy and/or pulmonary surgery, we were not able to investigate the possible separate pulmotoxic effects of these chemotherapeutics.

Twenty-seven out of 34 CCSs (79.4\%) with restrictive pulmonary function impairment had a decreased $\mathrm{D}_{\mathrm{L}} \mathrm{CO}$ but a normal $\mathrm{KCO}$. This type of diffusion capacity abnormality is indicative of a loss of lung volume or extra-pulmonary factors, such as underdevelopment of the thorax. Of these patients, 23 were treated with pulmonary radiotherapy, eight of whom also underwent surgery. Since surgery only involved metastasectomy in seven patients, surgery does not appear to explain the lower lung volumes observed in these patients. It may be hypothesised that pulmonary radiotherapy limits the development of new alveoli by limiting the ability of cells to proliferate or by interfering with vascular supply, and affects the development of the bony parts of the chest, which may explain these pulmonary function impairments. Moreover, only one CCS with restrictive pulmonary function impairment, treated with radiotherapy only, had both a decreased $\mathrm{D}_{\mathrm{L}} \mathrm{CO}$ and $\mathrm{KCO}$ suggesting increased vascular impairment that could be explained merely by loss of lung volume. Therefore, a vascular impairment disproportionate to the loss of lung volume, as reflected by a reduction in KCO due to therapy-induced damage to the alveocapillary membrane, appeared not to be a major problem in our cohort.

Bleomycin is the most commonly recognised cause of pulmonary function impairment in CCSs. However, the exact threshold dose for developing pulmonary function impairment after treatment with bleomycin is unclear. Cumulative doses $>450 \mathrm{mg} / \mathrm{m}^{2}$ were reported to be associated with a higher risk of pulmotoxicity, although doses as low as $20 \mathrm{mg} / \mathrm{m}^{2}$ may also induce pulmonary function impairment. ${ }^{724}$ We observed limited pulmonary function impairment in CCSs treated with bleomycin only. This may be explained by the fact that the median bleomycin dose was relatively low $\left(60 \mathrm{mg} / \mathrm{m}^{2}\right)$. Only four CCSs were treated with a bleomycin dose $>300 \mathrm{mg} / \mathrm{m}^{2}$, of whom two developed pulmonary function impairments.

Since exercise intolerance in CCSs is most often multicausal in nature, with pulmonary function impairment being one possible factor, we did not study symptoms experienced and limitations. CCSs are prone to a high rate of concurrent illness ${ }^{3} 4$ and may, apart from pulmonary function impairment, suffer from additional problems that may contribute to exercise intolerance (eg, chemotherapy-induced congestive heart failure). To discriminate between exercise intolerance due merely to pulmonary function impairment and/or other organ dysfunctions would have involved additional diagnostic procedures, including exercise testing. These procedures were not part of our routine followup. By definition, however, an $\mathrm{FEV}_{1}<80 \%$, and a TLC, FVC,

Table 3 Risk factors for pulmonary function impairment (grade 2 or higher)*

\begin{tabular}{|c|c|c|c|c|}
\hline \multirow[b]{2}{*}{ Risk factor } & \multicolumn{2}{|c|}{ Restrictive pulmonary function impairment } & \multicolumn{2}{|c|}{ Diffusion capacity impairment $\left(D_{L} C 0\right)$} \\
\hline & OR (95\% CI) & p Value & OR (95\% CI) & p Value \\
\hline \multicolumn{5}{|l|}{ Model 1} \\
\hline Sex, female versus male & $1.14(0.48$ to 2.70$)$ & 0.76 & $1.71(0.89$ to 3.26$)$ & 0.11 \\
\hline Age at diagnosis, years & $1.01(0.91$ to 1.12$)$ & 0.84 & $0.95(0.87$ to 1.03$)$ & 0.18 \\
\hline Follow-up duration, years & $1.02(0.95$ to 1.09$)$ & 0.58 & $1.02(0.97$ to 1.08$)$ & 0.38 \\
\hline Bleomycin, yes versus no & $1.50(0.38$ to 5.97$)$ & 0.57 & 1.99 (0.56 to 7.07$)$ & 0.29 \\
\hline Radiotherapy, yes versus no & 12.87 (3.37 to 49.08$)$ & 0.000 & $5.84(1.88$ to 18.14$)$ & 0.002 \\
\hline Surgery, yes versus no & $3.79(1.25$ to 11.50$)$ & 0.019 & 1.98 (0.68 to 5.75$)$ & 0.21 \\
\hline HD cyclophosphamide, yes versus no & $2.15(0.80$ to 5.79$)$ & 0.13 & $1.25(0.58$ to 2.71$)$ & 0.57 \\
\hline \multicolumn{5}{|l|}{ Model $2 \dagger$} \\
\hline Sex, female versus male & $1.11(0.46$ to 2.65$)$ & 0.82 & $1.69(0.89$ to 3.24$)$ & 0.11 \\
\hline Age at diagnosis, years & $1.02(0.92$ to 1.13$)$ & 0.75 & $0.95(0.87$ to 1.03$)$ & 0.19 \\
\hline Follow-up duration, years & $1.02(0.96$ to 1.09$)$ & 0.50 & $1.03(0.97$ to 1.08$)$ & 0.37 \\
\hline \multicolumn{5}{|l|}{ Therapy subgroup } \\
\hline Bleomycin only & 1 (Reference) & & 1 (Reference) & \\
\hline Radiotherapy only & $6.99(2.27$ to 21.54$)$ & 0.001 & $2.85(1.32$ to 6.19$)$ & 0.008 \\
\hline Surgery only & $1.59(0.15$ to 16.35$)$ & 0.70 & $1.03(0.23$ to 4.68$)$ & 0.97 \\
\hline Bleomycin with radiotherapy & 9.41 (1.71 to 51.86$)$ & 0.010 & $6.17(1.37$ to 27.84$)$ & 0.018 \\
\hline Bleomycin with surgery & - & 0.99 & $1.38(0.10$ to 18.66$)$ & 0.81 \\
\hline Radiotherapy with surgery & 33.44 (7.81 to 143.09$)$ & 0.000 & $5.98(1.64$ to 21.81$)$ & 0.007 \\
\hline HD cyclophosphamide, yes versus no & $2.10(0.78$ to 0.62$)$ & 0.14 & $1.25(0.57$ to 2.73$)$ & 0.58 \\
\hline
\end{tabular}

Note that all patients were treated with potentially pulmotoxic therapy.

*According to the Common Terminology Criteria for Adverse Events v3.0.

†In model 2, mutually adjusted $\mathrm{ORs}$ are shown.

$D_{L} C O$, carbon monoxide diffusion capacity corrected for haemoglobin; HD, high-dose. 
$\mathrm{D}_{\mathrm{L}} \mathrm{CO}$ and $\mathrm{KCO}<75 \%$ (grade 2 ) can be considered abnormal, reflecting physiological impairment. In addition, a predicted value $<60 \%$ in either $\mathrm{FEV}_{1}$, TLC, FVC, $\mathrm{D}_{\mathrm{L}} \mathrm{CO}$ or $\mathrm{KCO}$ can be considered proportionate to a $10-25 \%$ functional impairment. Clinically, these CCSs will have a $10-25 \%$ reduction in general functioning as represented by a decrease in the ability to perform daily life activities. A TLC or FVC $<50 \%$ and a $\mathrm{FEV}_{1}, \mathrm{D}_{\mathrm{L}} \mathrm{CO}$ or $\mathrm{KCO}<40 \%$ are proportionate to a $51-100 \%$ functional impairment. ${ }^{21}$

Our study has some limitations. As our study population consisted of CCSs treated in different eras, cohort effects might have been of influence on our study estimates. However, when performing a subgroup analysis on CCSs treated before and after 1985 there was no indication that our results were biased, since the effects of pulmotoxic therapy on pulmonary function impairment did not differ between CCSs treated in earlier and more recent eras. Moreover, we do not have baseline pulmonary function tests before the onset of childhood cancer. Consequently, it is impossible to evaluate which part of the pulmonary function impairment was caused by the cancer treatment. In addition, we did not investigate the longitudinal development of pulmonary function impairment. The natural history of pulmonary function impairment has not been well recognised. Recently, Inaba et al $l^{9}$ investigated the pattern of pulmonary dysfunction over time in 89 CCSs. They showed that pulmonary function progressively declined. However, this study only included patients treated with allogeneic haematopoietic stem cell transplantation for childhood haematological malignancies. Moreover, we were not able to take detailed radiation dosimetry into account, including the volume of the lungs irradiated and the dose to different parts of the lungs. Finally, we did not take smoking status into consideration, since we do not have precise and accurate data on smoking frequency and duration.

In conclusion, this cohort study shows that the prevalence of pulmonary function impairment in long-term adult CCSs is high. This may have long-term clinical consequences as these patients have a long life expectancy. During their lifetime, CCSs might be exposed to additional health risks, such as smoking, pollution and infections. Bleomycin, pulmonary radiotherapy and pulmonary surgery are all associated with pulmonary function impairment. Pulmonary radiotherapy, especially in combination with bleomycin or surgery, is the most important risk factor. The Dutch guidelines advise performing a pulmonary function test in CCSs treated with bleomycin, pulmonary radiotherapy and/or pulmonary surgery 5 and 10 years after diagnosis. ${ }^{25}$ Since we do not know if pulmonary function will worsen over time, individualised education and prevention appear to be justified. For example, for CCSs with asymptomatic pulmonary function impairment, influenza vaccination may be recommended. Future studies should focus on the evaluation of pulmonary function over time in CSSs treated with pulmotoxic therapy, and on the influence of radiotherapy dose and the extent of pulmonary surgery. Based upon these studies, decisions can be made about the optimal interval and duration of follow-up of pulmonary function in high-risk CCSs.

Acknowledgements The authors would like to thank R.C. Heinen for helping in identifying all eligible patients and in obtaining the data.

Funding This study was supported by the Foundation of Paediatric Cancer Research (SKK), Amsterdam, The Netherlands. The sponsor had no involvement in the design and execution of the study, in writing the manuscript and in the decision to submit it for publication.

\section{Competing interests None.}

Ethics approval The EKZ/AMC institutional review board reviewed and approved the collection of data used for the analyses presented.

Contributors RLM collected data, wrote the statistical analysis plan, cleaned and analysed the data, and drafted and revised the paper. NMT designed the study protocol, collected data, and drafted and revised the paper. HvdP designed the study protocol, collected data, wrote the statistical analysis plan, contributed to the interpretation of the results and revised the paper. PB and LCMK designed the study protocol, wrote the statistical analysis plan, contributed to the interpretation of the results and revised the paper. WH designed the study protocol, collected data and revised the paper. CCEK, FO, HAH and HNC contributed to the interpretation of the results and revised the paper.

Provenance and peer review Not commissioned; externally peer reviewed.

\section{REFERENCES}

1. Curry HL, Parkes SE, Powell JE, et al. Caring for survivors of childhood cancers: the size of the problem. Eur J Cancer 2006;42:501-8.

2. Gatta G, Zigon G, Capocaccia R, et al. Survival of European children and young adults with cancer diagnosed 1995-2002. Eur J Cancer 2009;45:992-1005.

3. Oeffinger KC, Mertens AC, Sklar CA, et al. Chronic health conditions in adult survivors of childhood cancer. N Engl J Med 2006;355:1572-82.

4. Geenen MM, Cardous-Ubbink MC, Kremer LC, et al. Medical assessment of adverse health outcomes in long-term survivors of childhood cancer. JAMA 2007;297:2705-15

5. Liles A, Blatt J, Morris D, et al. Monitoring pulmonary complications in long-term childhood cancer survivors: guidelines for the primary care physician. Cleve Clin J Med 2008;75:531-9.

6. Abid SH, Malhotra V, Perry MC. Radiation-induced and chemotherapy-induced pulmonary injury. Curr Opin Oncol 2001;13:242-8.

7. Hinkle AS, Proukou C, Chen Y. Pulmonary effects of antineoplastic therapy. In: Schwartz CL, Hobbie WL, Constine LS, et al, eds. Survivors of Childhood and Adolescent Cancer: A Multidisciplinary Approach. 2nd edn. New York: Springer, 2005:161-80.

8. Mertens AC, Yasui Y, Neglia JP, et al. Late mortality experience in five-year survivors of childhood and adolescent cancer: the Childhood Cancer Survivor Study. J Clin Oncol 2001;19:3163-72.

9. Inaba H, Yang J, Pan J, et al. Pulmonary dysfunction in survivors of childhood hematologic malignancies after allogeneic hematopoietic stem cell transplantation. Cancer 2010;116:2020-30.

10. Oguz A, Tayfun T, Citak EC, et al. Long-term pulmonary function in survivors of childhood Hodgkin disease and non-Hodgkin lymphoma. Pediatr Blood Cancer 2007:49:699-703.

11. Bossi G, Cerveri I, Volpini E, et al. Long-term pulmonary sequelae after treatment of childhood Hodgkin's disease. Ann Oncol 1997;8(Suppl 1):19-24.

12. Fulgoni $\mathbf{P}$, Zoia MC, Corsico $\mathrm{A}$, et al. Lung function in survivors of childhood acute lymphoblastic leukemia. Chest 1999;116:1163-7.

13. Hoffmeister PA, Madtes DK, Storer BE, et al. Pulmonary function in long-term survivors of pediatric hematopoietic cell transplantation. Pediatr Blood Cancer 2006;47:594-606.

14. Nysom K, Holm K, Hertz $\mathrm{H}$, et al. Risk factors for reduced pulmonary function after malignant lymphoma in childhood. Med Pediatr Oncol 1998;30:240-8.

15. Nysom K, Holm K, Olsen JH, et al. Pulmonary function after treatment for acute lymphoblastic leukaemia in childhood. Br J Cancer 1998;78:21-7.

16. Fanfulla $\mathbf{F}$, Locatelli $\mathrm{F}$, Zoia $\mathrm{MC}$, et al. Pulmonary complications and respiratory function changes after bone marrow transplantation in children. Eur Respir $\mathrm{J}$ 1997:10:2301-6.

17. Kaplan $\mathbf{E}$, Sklar C, Wilmott R, et al. Pulmonary function in children treated for rhabdomyosarcoma. Med Pediatr Oncol 1996;27:79-84.

18. Mertens AC, Yasui Y, Liu Y, et al. Pulmonary complications in survivors of childhood and adolescent cancer. A report from the Childhood Cancer Survivor Study. Cancer 2002:95:2431-41.

19. From the Global Strategy for the Diagnosis, Management and Prevention of COPD. Global Initiative for Chronic Obstructive Lung Disease (GOLD), 2008. http://www. goldcopd.org/.

20. National Cancer Institute. CTEP. CTCAE v3.0. NCI, 2003.

21. Cocchiarella L, Andersson GBJ. Guides to the Evaluation of Permanent Impairment 5th edn. Chicago, III. American Medical Association Press, 2001.

22. Benoist MR, Lemerle J, Jean R, et al. Effects of pulmonary function of whole lung irradiation for Wilm's tumour in children. Thorax 1982;37:175-80.

23. Weiner DJ, Maity A, Carlson CA, et al. Pulmonary function abnormalities in children treated with whole lung irradiation. Pediatr Blood Cancer 2006;46:222-7.

24. Blum RH, Carter SK, Agre K. A clinical review of bleomycin-a new antineoplastic agent. Cancer 1973;31:903-14.

25. Dutch Childhood Oncology Group. Richtlijn follow-up na kinderkanker meer dan 5 jaar na diagnose. Den Haag/Amsterdam: SKION, 2010. http://www.skion.nl/. 